PROCEEDINGS OF THE

AMERICAN MATHEMATICAL SOCIETY

Volume 129, Number 11, Pages 3143-3149

S 0002-9939(01)05987-1

Article electronically published on April 16, 2001

\title{
ON THE HOMOLOGY OF SPLIT EXTENSIONS WITH $p$-ELEMENTARY KERNEL
}

\author{
MARCIN CHALUPNIK
}

(Communicated by Ralph Cohen)

\begin{abstract}
We study a Hochschild-Serre spectral sequence associated to a split group extension with kernel $(\mathbf{Z} / p)^{n}$. It is shown that a large part of $E_{2}^{0 *}$ must survive to infinity. We also sketch the general procedure of computing this surviving group.
\end{abstract}

\section{INTRODUCTION}

It is often useful to decompose a spectral sequence into eigenspaces of an automorphism of the sequence. In the case of a Hochschild-Serre spectral sequence associated to a split extension with abelian kernel the Liebermann trick (see [Sa], p. 262 ) provides an important example of this. One takes the automorphism induced by multiplication by a scalar in the kernel of the extension. For example, it is easy using this method to show that in a split extension with abelian kernel

$$
0 \rightarrow A \rightarrow H \rightarrow G \rightarrow 1
$$

we have $H_{i}(H, \mathbf{Q})=\bigoplus_{0 \leq j \leq i}\left(H_{i-j}\left(G, \Lambda^{j}(A \otimes \mathbf{Q})\right)\right)$. If one considers the homology with $\mathbf{F}_{p}$-coefficients, the situation becomes more involved. The first problem is that scalars have only finite multiplicative order and the second is that the homology of an abelian group also contains a part generated by elements of degree 2 (for $p$ odd). For these reasons a Hochschild-Serre spectral sequence can have many nontrivial differentials and is generally hard to understand.

In the present paper we use the Liebermann trick together with an analysis of a scalar extension to show triviality of some differentials when one takes $\mathbf{F}_{p^{-}}$ coefficients. We apply this technique in Section 2 to show that a part of the 0 -th column survives which is close to $\Lambda^{*}(A)_{G}$. In Section 3 we discuss some examples, in particular we show that $\Lambda^{*}(A)_{G}$ does not always embed into $H_{*}\left(H, \mathbf{F}_{p}\right)$.

It is a pleasure to thank Stanislaw Betley for many interesting discussions and strong encouragement.

\section{THE THEOREM}

Let

$$
0 \rightarrow A \rightarrow H \rightarrow G \rightarrow 1
$$

Received by the editors August 25, 1999 and, in revised form, March 8, 2000.

2000 Mathematics Subject Classification. Primary 20J06.

The author was partially supported by the Polish scientific grant (KBN) 2 P03A 01113.

(C)2001 American Mathematical Society 
be a split extension with $A=(\mathbf{Z} / p)^{n}$ (we specialize to this case only to simplify notation, for general (abelian) $A$ our Theorem 1 remains true if we replace $A$ by $A \otimes_{\mathbf{Z}} \mathbf{F}_{p}$ ). We will consider a homological Hochschild-Serre spectral sequence with $\mathbf{F}_{p}$-coefficients corresponding to this extension. Denoting by $D^{j}$ a $j$-th divided power we have the natural identification of the $E^{2}$-term:

$$
\begin{array}{cc}
E_{i j}^{2}=\bigoplus_{k+2 l=j} H_{i}\left(G, \Lambda^{k}(A) \otimes D^{l}(A)\right) & \text { for } p \text { odd, } \\
E_{i j}^{2}=H_{i}\left(G, D^{j}(A)\right) & \text { for } p=2
\end{array}
$$

(unless otherwise stated $\otimes$ means $\otimes_{\mathbf{F}_{p}}$ ).

Let us define $H_{i}\left(G, \Lambda^{*}(A)\right)_{\text {reg }}$ to be

$$
H_{i}\left(G, \Lambda^{*}(A)\right) / \operatorname{ker}\left(H_{i}\left(G, \Lambda^{*}(A)\right) \rightarrow H_{i}\left(G, A^{\otimes *}\right)\right)
$$

where the arrow is induced by the natural embedding $\Delta: \Lambda^{*}(A) \hookrightarrow A^{\otimes *}$. Similarly, we put

$$
H_{i}\left(G, D^{*}(A)\right)_{r e g}=H_{i}\left(G, D^{*}(A)\right) / \operatorname{ker}\left(H_{i}\left(G, D^{*}(A)\right) \rightarrow H_{i}\left(G, A^{\otimes *}\right)\right)
$$

for $\Gamma: D^{*}(A) \hookrightarrow A^{\otimes *}$.

In our spectral sequence only some pieces of the groups we are interested in survive, hence we should carefully differ between $E^{2}$ and the higher $E^{r}$. Thus, in order to make the formulation of Theorem 1 clear and to avoid a confusion in its proof we introduce some notation. For $i \leq 1$ we have natural epimorphisms $\alpha_{i j}^{r}: E_{i j}^{2} \rightarrow E_{i j}^{r}$. Then we put $B_{i j}=\bigcup_{r \geq 2} \operatorname{ker}\left(\alpha_{i j}^{r}\right) \cap H_{i}\left(G, \Lambda^{j}(A)\right)$ for $p$ odd and $B_{i j}=\bigcup_{r \geq 2} \operatorname{ker}\left(\alpha_{i j}^{r}\right) \cap H_{i}\left(G, D^{j}(A)\right)$ for $p=2$.

Theorem 1. In the sequence (2) we have $B_{i j} \subset \operatorname{ker}\left(\Delta_{*}\right)$ for $i \leq 1$. In other words, the spaces $H_{i}\left(G, \Lambda^{j}(A)\right)_{\text {reg }}$ for $i \leq 1$ survive to infinity. The same holds for the sequence (3) when we replace $\Lambda^{j}(A)$ by $D^{j}(A)$.

Proof. We begin with some remarks concerning functoriality of semidirect products. It is well known that the class of spilt extensions of a fixed group $G$ by abelian kernels is in bijection with the class of $\mathbf{Z}[G]$-modules via construction of semidirect product. Moreover, this assignment yields an isomorphism of the category of $\mathbf{Z}[G]$-modules and the category of split extensions of $G$ with abelian kernels where morphisms are morphisms of extensions being identity on $G$. The practical consequence is that any $G$-homomorphism between kernels of two extensions induces a morphism of spectral sequences.

The idea of the proof (for $p$ odd) is as follows. We look at the automorphism of the spectral sequence (2) induced by the $G$-automorphism of $A$ defined by the formula $x \mapsto c x$ for a given scalar $c \in \mathbf{F}_{p}{ }^{*}$ (we will frequently use the structure of $\mathbf{F}_{p}$-linear space on $A$ ). Then it is easy to see that the space

$$
H_{*}\left(G, \Lambda^{k}(A) \otimes D^{l}(A)\right)
$$

belongs to the eigenspace of the induced automorphism for the eigenvalue $c^{k+l}$ and that the whole spectral sequence is a direct sum of eigensequences for eigenvalues $1, c, c^{2}, \ldots, c^{p-1}$. At this point it is clear for example that there are no differentials coming to $H_{*}\left(G, \Lambda^{k}(A)\right)$ for $k<p$ because all $E_{i j}^{*}$ for $j<k$ belong to eigenspaces of $c^{s}$ for $s<k$ and differentials must preserve the decomposition. Thus $H_{0}\left(G, \Lambda^{k}(A)\right)$ and $H_{1}\left(G, \Lambda^{k}(A)\right)$ for $k<p$ survives. Unfortunately, this argument fails for $k \geq p$ 
since $c^{p}=c$ for any $c \in \mathbf{F}_{p}{ }^{*}$. We partially overcome this difficulty comparing the sequence (2) with a sequence associated to a split extension with kernel equipped with $G$-automorphism of higher order.

Therefore let us consider a split extension

$$
0 \rightarrow A \otimes L \rightarrow H(L) \rightarrow G \rightarrow 1
$$

where $L$ is a one-dimensional space over a field $\mathbf{F}_{q}$ with $q=p^{d}$ elements regarded as a trivial $G$-module. We shall describe ${ }^{\prime} E^{2}$ - the second page of a Hochschild-Serre spectral sequence (with coefficients in $\mathbf{F}_{q}$ ) associated to it. We focus here on the case when $p$ is odd. We should take into account the $\mathbf{F}_{q}$-structure appearing in this new sequence. More precisely, we describe ${ }^{\prime} E_{* *}^{2}$ as evaluations on $L$ of functors from the category of finite $\mathbf{F}_{q}$-spaces to itself assigning to a $\mathbf{F}_{q}$-space $V$ the entries in the spectral sequence associated to the extension

$$
0 \rightarrow A \otimes V \rightarrow H(V) \rightarrow G \rightarrow 1
$$

According to a functorial description of the homology of an abelian group (see e.g. $\mathrm{Qu} 2$, p. 210) and the natural isomorphism $V \otimes \mathbf{F}_{q}=\bigoplus_{t=0}^{d-1} V^{(t)}$ (here $V^{(t)}$ means the space $V$ with $\mathbf{F}_{q}$-structure twisted by $t$-th Frobenius) we get

(4)

$$
\begin{aligned}
{ }^{\prime} E_{i j}^{2} & =\bigoplus_{k+2 l=j} H_{i}\left(G, \Lambda^{k}\left(A \otimes L \otimes \mathbf{F}_{q}\right) \otimes \mathbf{F}_{q} D^{l}\left(A \otimes L \otimes \mathbf{F}_{q}\right)\right) \\
& =\bigoplus_{k+2 l=j} H_{i}\left(G, \Lambda^{k}\left(\bigoplus_{t=0}^{d-1} A \otimes L^{(t)}\right) \otimes \mathbf{F}_{q} D^{l}\left(\bigoplus_{t=0}^{d-1} A \otimes L^{(t)}\right)\right) \\
& =\bigoplus_{k+2 l=j \sum k_{t}=k \sum l_{t}=l} \bigoplus_{i} H_{i}\left(G, \Lambda^{k_{0}}\left(A \otimes L^{(0)}\right) \otimes_{\mathbf{F}_{q}} \ldots \otimes_{\mathbf{F}_{q}} \Lambda^{k_{d-1}}\left(A \otimes L^{(d-1)}\right)\right. \\
& \left.\otimes_{\mathbf{F}_{q}} D^{l_{0}}\left(A \otimes L^{(0)}\right) \otimes_{\mathbf{F}_{q}} \ldots \otimes_{\mathbf{F}_{q}} D^{l_{d-1}}\left(A \otimes L^{(d-1)}\right)\right)
\end{aligned}
$$

(we alert the reader that in these formulas exterior and divided powers are taken over $\mathbf{F}_{q}$ ). Now take a scalar $c \in \mathbf{F}_{q}{ }^{*}$ of multiplicative order $p^{d}-1$. We define the $G$-automorphism of $A \otimes L$ by the formula $a \otimes x \mapsto a \otimes c x$. In order to understand the induced automorphism of the spectral sequence (4) observe that multiplication by $c$ on $L$ induces on $A \otimes L^{(t)}$ multiplication by $c^{p^{t}}$. Thus the space

$$
H_{*}\left(G, \bigotimes_{t=0}^{d-1} \Lambda^{k_{t}}\left(A \otimes L^{(t)}\right) \otimes \mathbf{F}_{q} \bigotimes_{t=0}^{d-1} D^{l_{t}}\left(A \otimes L^{(t)}\right)\right)
$$

(big tensor products are over $\mathbf{F}_{q}$ ) belongs to the eigenspace of the eigenvalue $c^{\sum_{t=0}^{d-1}\left(k_{t}+l_{t}\right) p^{t}}$. The crucial fact is that here exponents in eigenvalues are taken modulo $p^{d}-1$ hence more differentials must be trivial than in sequence (2). So our next task will be to compare both spectral sequences. Before doing this however, we introduce some notation. For a sequence of nonnegative integers $\mathbf{k}=\left(k_{0}, \ldots, k_{d-1}\right)$ we define $r(\mathbf{k})$ to be the number $r(\mathbf{k})=\sum_{t} k_{t}$ and $|\mathbf{k}|$ to be $\sum_{t} k_{t} p^{t}\left(\bmod p^{d}-1\right)$. The following elementary arithmetic lemma holds

Lemma 1. Let $0 \leq j<d(p-1)$. Then there exists $\mathbf{k}$ and a number a such that $r(\mathbf{k})=j,|\mathbf{k}|=a$ and $\left|\mathbf{k}^{\prime}\right| \neq a$ for any $\mathbf{k}^{\prime}$ such that $r\left(\mathbf{k}^{\prime}\right) \leq j$. 
Proof. Let $j=f(p-1)+g$ where $g<p-1$. We put

$$
k_{i}= \begin{cases}0 & \text { for } \quad i<d-f-1, \\ g & \text { for } i=d-f-1, \\ p-1 & \text { for } \quad d-f-1<i \leq d-1 .\end{cases}
$$

We are going to show that for any $\mathbf{k}^{\prime}$ satisfying $\left|\mathbf{k}^{\prime}\right|=|\mathbf{k}|$ we have $r\left(\mathbf{k}^{\prime}\right)>r(\mathbf{k})$. Let us take such $\mathbf{k}^{\prime}$. If there exists $k_{i_{0}}^{\prime} \geq p$, we may replace $\mathbf{k}^{\prime}$ by $\mathbf{k}^{\prime \prime}$ having the same || but smaller $r$ defining

$$
k_{i}^{\prime \prime}= \begin{cases}k_{i}^{\prime}-p & \text { for } i=i_{0}, \\ k_{i+1}^{\prime}+1 & \text { for } i=i_{0}+1, \\ k_{i}^{\prime} & \text { for } i \neq i_{0}, i_{0}+1\end{cases}
$$

(we use here the convention $k_{d}=k_{0}$ ). Thus, iterating this procedure, we may assume that all $k_{i}^{\prime}$ are smaller than $p$. But in this case the only possibility for $\left|\mathbf{k}^{\prime}\right|=|\mathbf{k}|$ is $\mathbf{k}^{\prime}=\mathbf{k}$.

Let $\Lambda^{\mathbf{k}}(A \otimes L)$ denote $\Lambda^{k_{0}}(A \otimes L) \otimes \ldots \otimes \Lambda^{k_{d-1}}\left(A \otimes L^{(d-1)}\right)$. If for $j<$ $d(p-1)$ we take $\mathbf{k}$ as in Lemma 1 , then there cannot be any differentials coming to $H_{*}\left(G, \Lambda^{\mathbf{k}}(A \otimes L)\right)$ (and its subspaces in the higher $\left.{ }^{\prime} E^{r}\right)$. Thus the spaces $H_{i}\left(G, \Lambda^{\mathbf{k}}(A \otimes L)\right)$ for $i \leq 1$ survive in sequence (4).

We now want to construct a morphism from the spectral sequence (2) to (4). First we should replace (2) by (5)-its counterpart with $\mathbf{F}_{q}$-coefficients. In this new sequence we have

$$
{ }^{\prime \prime} E_{i j}^{2}=\bigoplus_{k+2 l=j} H_{i}\left(G, \Lambda^{k}\left(A \otimes \mathbf{F}_{q}\right) \otimes_{\mathbf{F}_{q}} D^{l}\left(A \otimes \mathbf{F}_{q}\right)\right) .
$$

There is a morphism $\Phi$ from (2) to (5) which is, by the Kunneth formula, on $E^{2}$ just induced by scalar extension in all tensors appearing as the coefficients of the homology of $G$. Now to obtain a morphism from (5) to (4) it suffices to choose a $G$-homomorphism from $A$ to $A \otimes L$ which is possible since $L$ is a trivial $G$-module. In order to make formulas explicit let us identify $L$ with $\mathbf{F}_{q}$. Then we take the homomorphism from $A$ to $A \otimes \mathbf{F}_{q}$ sending $a$ to $a \otimes 1$ and we will consider the morphism of spectral sequences $\Psi$ induced by this $G$-homomorphism. Under the isomorphism $\left(A \otimes \mathbf{F}_{q}\right) \otimes \mathbf{F}_{q}=\bigoplus_{t=0}^{d-1}\left(A \otimes \mathbf{F}_{q}{ }^{(t)}\right)$ the morphism $\Psi$ from (5) to (4) is induced on $E^{2}$ by the morphism of coefficients $\psi: A \otimes \mathbf{F}_{q} \rightarrow \bigoplus_{t=0}^{d-1}\left(A \otimes \mathbf{F}_{q}{ }^{(t)}\right)$ sending $a \otimes x$ to $\bigoplus_{t=0}^{d-1}\left(a \otimes x^{p^{t}}\right)$. We focus on the groups $H_{*}\left(G, \Lambda^{j}\left(A \otimes \mathbf{F}_{q}\right)\right)$. We would like to describe the map $\pi_{\mathbf{k}} \circ \psi_{*}: H_{*}\left(G, \Lambda^{j}\left(A \otimes \mathbf{F}_{q}\right)\right) \rightarrow H_{*}\left(G, \Lambda^{\mathbf{k}}\left(A \otimes \mathbf{F}_{q}\right)\right)$ where for given a sequence $\mathbf{k}$ with $r(\mathbf{k})=j$ the map $\pi_{\mathbf{k}}$ is the projection from ${ }^{\prime} E_{* j}^{2}$ onto $H_{*}\left(G, \Lambda^{\mathbf{k}}\left(A \otimes \mathbf{F}_{q}\right)\right)$. According to the above formulas, $\pi_{\mathbf{k}} \circ \psi_{*}$ may be factorized as $f_{*} \circ \mathrm{com}_{*}$ where

$$
\operatorname{com}_{*}: H_{*}\left(G, \Lambda^{j}\left(A \otimes \mathbf{F}_{q}\right)\right) \rightarrow H_{*}\left(G, \Lambda^{k_{0}}\left(A \otimes \mathbf{F}_{q}\right) \otimes \ldots \otimes \Lambda^{k_{d-1}}\left(A \otimes \mathbf{F}_{q}\right)\right)
$$

is induced by the iterated comultiplication map in the $\mathbf{F}_{q}-$ Hopf algebra $\Lambda_{\mathbf{F}_{q}}^{*}\left(A \otimes \mathbf{F}_{q}\right)$ while

$$
\begin{aligned}
& f_{*}: H_{*}\left(G, \Lambda^{k_{0}}\left(A \otimes \mathbf{F}_{q}\right) \otimes \ldots \otimes \Lambda^{k_{d-1}}\left(A \otimes \mathbf{F}_{q}\right)\right) \\
& \quad \rightarrow H_{*}\left(G, \Lambda^{k_{0}}\left(A \otimes \mathbf{F}_{q}^{(0)}\right) \otimes \ldots \otimes \Lambda^{k_{d-1}}\left(A \otimes \mathbf{F}_{q}^{(d-1)}\right)\right)
\end{aligned}
$$

is determined by the $G$-isomorphism $f_{k_{0}} \otimes \ldots \otimes f_{k_{d-1}}$ defined on a factor $\Lambda^{k_{t}}$ by the formula $f_{k_{t}}(a \otimes x)=a \otimes x^{p^{t}}$. 
Now we are in a position to prove the theorem. Given $x \in H_{i}\left(G, \Lambda^{j}(A)\right)(i \leq 1)$ belonging to $\operatorname{ker}\left(\alpha_{i j}^{r}\right)$, choose $\mathbf{k}$ as in Lemma 1 and consider the commutative diagram

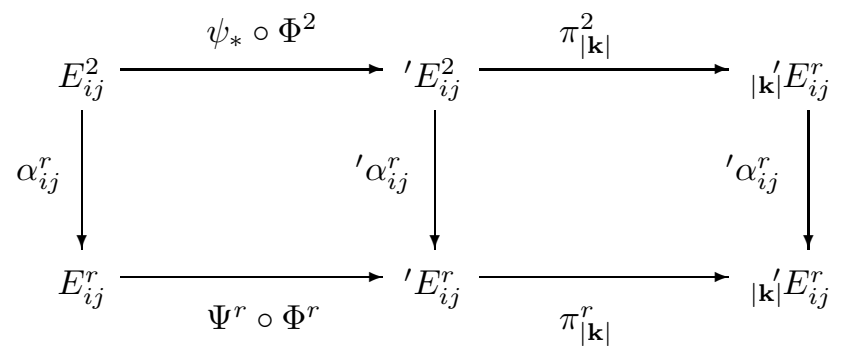

where $|\mathbf{k}| E$ denotes the subsequence corresponding to the eigenvalue $|\mathbf{k}|$ and $\pi_{|\mathbf{k}|}$ is a natural projection (it is a morphism of spectral sequences in contrast to $\pi_{\mathbf{k}}$ ). Now suppose that

$$
{ }^{\prime} \alpha_{i j}^{r} \circ \pi_{|\mathbf{k}|}^{2} \circ \psi_{*} \circ \Phi^{2}(x)=0 .
$$

Since $\pi_{|\mathbf{k}|}^{2} \circ \psi_{*} \circ \Phi^{2}(x)=\pi_{\mathbf{k}} \circ \psi_{*} \circ \Phi^{2}(x) \in H_{i}\left(G, \Lambda^{\mathbf{k}}\left(A \otimes \mathbf{F}_{q}\right)\right)$, which by the paragraph after Lemma 1 survives to infinity, we thus obtain

$$
\pi_{\mathbf{k}} \circ \psi_{*} \circ \Phi^{2}(x)=0 .
$$

Now by identifications we have worked out earlier we get

$$
0=\pi_{\mathbf{k}} \circ \psi_{*} \circ \Phi^{2}(x)=f_{*} \circ \operatorname{com}_{*} \circ \Phi^{2}(x) .
$$

But since $f_{*}$ is an isomorphism, we have

$$
\mathrm{com}_{*} \circ \Phi^{2}(x)=0 .
$$

At last, by the Kunneth formula, $\operatorname{ker}\left(\operatorname{com}_{*} \circ \Phi^{2}\right)=\operatorname{ker}\left(\mathrm{com}_{*}^{\prime}\right)$ where $\operatorname{com}^{\prime}$ is iterated comultiplication in $\mathbf{F}_{p}-$ Hopf algebra $\Lambda_{\mathbf{F}_{p}}^{*}(A)$. Thus we get that $\operatorname{com}_{*}^{\prime}(x)=0$. Since $\Delta$ is also iterated comultiplication (corresponding to the partition $(1, \ldots, 1)$ ), then $\Delta$ factors through $\mathrm{com}^{\prime}$ and we obtain $\operatorname{ker}\left(\operatorname{com}_{*}^{\prime}\right) \subset \operatorname{ker}\left(\Delta_{*}\right)$ concluding the proof. We note that in fact $\operatorname{ker}\left(\operatorname{com}_{*}^{\prime}\right)=\operatorname{ker}\left(\Delta_{*}\right)$, and we have introduced $\Delta$ only in order to simplify the statement of the theorem, since com depends on $j$ in a more complicated way.

For the case $p=2$ we proceed analogously. The only difference is the different description of the homology of an abelian group which does not affect our arguments.

\section{REMARKS AND EXAMPLES}

This paper was motivated by the following example. We consider a split extension of finite $\mathbf{F}_{p}$-algebras

$$
\mathbf{F}_{p} \rightarrow \mathbf{F}_{p}[x] / x^{2} \rightarrow \mathbf{F}_{p}
$$

This extension induces a split group extension

$$
0 \rightarrow M(J) \rightarrow G L(R) \rightarrow G L(S) \rightarrow 1
$$


where $G L$ is the colimit of general linear groups, $M$ is the colimit of additive groups of matrices and $G L\left(\mathbf{F}_{p}\right)$ acts on $M\left(\mathbf{F}_{p}\right)$ by conjugation (of course, a group extension exists already at the level of $G L_{n}$ and $M_{n}$ ). It was shown by Goodwillie that for any split extension of rings $J \rightarrow R \rightarrow S$, where $J$ is a free $S$-bimodule regarded as an ideal with trivial multiplication, that $\Lambda^{*}(M(J) \otimes \mathbf{Q})_{G L(S)}=H_{*}(F, \mathbf{Q})$ where $F$ is the homotopy fiber of the induced map $B G L^{+}(R) \rightarrow B G L^{+}(S)$ ([Go], p. 395). This result awakened my interest to the space $\Lambda^{*}(A)_{G}$. For example, if Goodwillie's theorem was also true with coefficients in $\mathbf{F}_{p}$, then thanks to $\widetilde{H}_{*}\left(G L\left(\mathbf{F}_{p}\right), \mathbf{F}_{p}\right)=0$ (Qu1]) we would obtain

$$
H_{*}\left(G L\left(\mathbf{F}_{p}[x] / x^{2}\right), \mathbf{F}_{p}\right)=\Lambda^{*}\left(M\left(\mathbf{F}_{p}\right), \mathbf{F}_{p}\right)_{G L\left(\mathbf{F}_{p}\right)} .
$$

It has been known since the early eighties (see e.g. [EF]) that this equality cannot hold because $H_{*}\left(G L\left(\mathbf{F}_{p}[x] / x^{2}\right), \mathbf{F}_{p}\right)$ is too big, but initially I conjectured that $\Lambda^{*}\left(M\left(\mathbf{F}_{p}\right), \mathbf{F}_{p}\right)_{G L\left(\mathbf{F}_{p}\right)}$ embeds into $H_{*}\left(G L\left(\mathbf{F}_{p}[x] / x^{2}\right), \mathbf{F}_{p}\right)$ through the edge homomorphism in the sequence (2) associated to the extension (7). This hope was destroyed by results of $[\mathrm{HM}]$. Hesselholt and Madsen have computed $K_{*}\left(\mathbf{F}_{p}[x] / x^{2}\right)$, but since $B G L^{+}\left(\mathbf{F}_{p}[x] / x^{2}\right) \hat{p}$ is a generalized Eilenberg-Mac Lane spectrum, it also determines $H_{*}\left(G L\left(\mathbf{F}_{p}[x] / x^{2}\right), \mathbf{F}_{p}\right)$. In particular, their formulas give

$$
H_{2}\left(G L\left(\mathbf{F}_{2}[x] / x^{2}\right), \mathbf{F}_{2}\right)=\mathbf{F}_{2},
$$

but it is easy to see that $H_{0}\left(G L\left(\mathbf{F}_{2}\right), D^{2}\left(M\left(\mathbf{F}_{2}\right)\right)\right)=\mathbf{F}_{2}{ }^{2}$. This shows that the spectral sequence (3) corresponding to the extension $\mathbf{F}_{2} \rightarrow \mathbf{F}_{2}[x] / x^{2} \rightarrow \mathbf{F}_{2}$ must have a nontrivial differential arriving at $H_{0}\left(G L\left(\mathbf{F}_{2}\right), D^{2}\left(M\left(\mathbf{F}_{2}\right)\right)\right)$. A similar example may be also constructed for $p=3$. Thus the restriction to $\left(H_{i}\right)_{r e g}$ in Theorem 1 is necessary.

We look more closely at the groups in Theorem 1 , and focus on $H_{0}\left(G, \Lambda^{*}(A)\right)_{\text {reg }}$ (for $p$ odd) which, as we have seen, appears in another context but is also more computable than $H_{1}\left(G, \Lambda^{*}(A)\right)_{\text {reg }}$. In general, the process of computing $H_{0}\left(G, \Lambda^{j}(A)\right)_{\text {reg }}$ divides into two steps. The first requires knowledge not only about $H_{0}(G, A)$ but also about the whole representation $G \rightarrow \operatorname{Aut}(A)$ to determine $H_{0}\left(G, A^{\otimes j}\right)$. The second is to describe the action of the group $\Sigma_{j}$ on $H_{0}\left(G, A^{\otimes j}\right)$ induced by permutation of factors in $A^{\otimes j}$. If one completes this program, in order to obtain a formula for $H_{0}\left(G, \Lambda^{j}(A)\right)_{\text {reg }}$ it suffices to observe that it may be identified with the image of the endomorphism $A l t_{*}$ of $H_{0}\left(G, A^{\otimes j}\right)$ given by the antisymmetrization formula

$$
A l t_{*}(x)=\sum_{\sigma \in \Sigma_{j}} \operatorname{sgn}(\sigma) \sigma . x
$$

(an analogous fact is not true for $p=2$ because $D^{j}(A)$ is not an image of $A^{\otimes j}$ ).

To illustrate this algorithm we apply it to extension (7). By the First Fundamental Theorem of (co)Invariant Theory $\mathrm{dCP}$ we have

$$
H_{0}\left(G L\left(\mathbf{F}_{p}\right), M\left(\mathbf{F}_{p}\right)^{\otimes j}\right)=\mathbf{F}_{p}\left[\Sigma_{j}\right]
$$

and the action of the symmetric group on the group algebra is given by the formula $\sigma . e_{\tau}=e_{\sigma \tau \sigma^{-1}}$. Now we should describe the image of the antisymmetrization map (8). Let us take $e_{\tau} \in \mathbf{F}_{p}\left[\Sigma_{j}\right]$ and consider two different cases: if the centralizer of $\tau$ contains an odd permutation, and if it does not. In the first case we have $\operatorname{Alt}_{*}\left(e_{\tau}\right)=0$ so we focus on the case when the centralizer consists of only even 
permutations. Then choosing representatives for $\Sigma_{j} / \operatorname{Centr}(\tau)$ we may write

$$
A l t_{*}\left(e_{\tau}\right)=|\operatorname{Centr}(\tau)| \cdot \sum_{\tau^{\prime} \in \Sigma_{j} / \operatorname{Centr}(\tau)} \operatorname{sgn}\left(\tau^{\prime}\right) \cdot e_{\tau^{\prime} \tau} .
$$

From this formula the following consequences may be immediately derived: $A l t_{*}\left(e_{\tau}\right)$ depends only on the conjugacy class of $\tau$, it is nontrivial if $\operatorname{Centr}(\tau)$ contains no odd permutation and is of order prime to $p$, elements in different conjugacy classes have images linearly independent. Using elementary combinatorics of the symmetric group we may translate these conditions into the language of partitions of $j$. The result is

$$
\begin{aligned}
\operatorname{dim}\left(H_{0}\left(G, \Lambda^{j}(A)\right)_{\text {reg }}\right)= & \{\text { the number of partitions of } j \text { into } \\
& \text { different odd numbers prime to } p\}
\end{aligned}
$$

We point out that the last requirement is nothing but the condition for regularity of a conjugacy class in the sense of representation theory. This explains our notation for $\left(H_{*}\right)_{\text {reg }}$.

We finish by making one disappointing remark concerning the group $H_{1}\left(G L\left(\mathbf{F}_{p}\right), \Lambda^{*}\left(M\left(\mathbf{F}_{p}\right)\right)\right)_{r e g}$. Namely, in contrast to $H_{0}\left(G L\left(\mathbf{F}_{p}\right), \Lambda^{j}\left(M\left(\mathbf{F}_{p}\right)\right)\right)_{r e g}$, it quite easily follows from [Be1] and [Be2] that $H_{1}\left(G L\left(\mathbf{F}_{p}\right), \Lambda^{j}(M(J))\right)=0$ for $p>2$ and $j<p$.

\section{REFERENCES}

[Be1] S. Betley, Calculations in THH-Theory, J. Algebra 180, (1996), 445-458. MR 97a:19002

[Be2] S. Betley, Stable K-Theory of Finite Fields, K-Theory 17 (1999), 103-111. MR 2000d:18006

[dCP] C. de Concini, C. Procesi, A characteristic free approach to invariant theory, Advances in Math. 21 (1976), no. 3, 330-354. MR 54:10305

[EF] L. Evens, E. Friedlander, On $K_{*}\left(Z / p^{2}\right)$ and related homology groups, Trans. Amer. Math. Soc. 270 (1982), 1-46. MR 83j:18013

[Go] T. Goodwillie, Relative algebraic K-theory and cyclic homology, Ann. Math. 124 (1986), 347-402. MR 88b:18008

[HM] L. Hesselholt, I. Madsen, On the K-theory of finite algebras over Witt vectors of perfect fields, Topology 36 (1997), 29-101. MR 97i:19002

[Qu1] D. Quillen, On the cohomology and $K$-theory of the general linear groups over a finite field, Ann. Math. 96 (1972), 552-586. MR 47:3565

[Qu2] D. Quillen, Characteristic classes of representations, Lect. Notes in Math. 551, (1976) (189-216). MR 58:28215

[Sa] C.-H. Sah, Cohomology of split extensions, J. Algebra 29 (1974), 255-302. MR 52:14083

Instytut Matematyki, University of Warsaw, ul. Banacha 2, 02-097 Warsaw, Poland

E-mail address: mchal@mimuw.edu.pl 